\title{
THE ORGANIZATION OF EXPERTISE IN THE PRESENCE OF COMMUNICATION
}

\author{
Flavia Roldán ${ }^{1}$
}

${ }^{1}$ Post-Doctoral Public-Private Sector Research Center, IESE 
The Public-Private Center is a Research Center based at IESE Business School. Its mission is to develop research that analyses the relationships between the private and public sectors primarily in the following areas: regulation and competition, innovation, regional economy and industrial politics and health economics.

Research results are disseminated through publications, conferences and colloquia. These activities are aimed to foster cooperation between the private sector and public administrations, as well as the exchange of ideas and initiatives.

The sponsors of the SP-SP Center are the following:

- Accenture

- Ajuntament de Barcelona

- ATM, FGC y TMB

- Caixa Manresa

- Cambra Oficial de Comerç, Indústria i Navegació de Barcelona

- Consell de l'Audiovisual de Catalunya

- Departamento de Economía y Finanzas de la Generalitat de Catalunya

- Departamento de Innovación, Universidades y Empresa de la Generalitat de Catalunya

- Diputació de Barcelona

- Endesa

- Fundació AGBAR

- Garrigues

- Mediapro

- Microsoft

- Sanofi Aventis

The contents of this publication reflect the conclusions and findings of the individual authors, and not the opinions of the Center's sponsors. 


\title{
The Organization of Expertise in the Presence of Communication
}

\author{
Flavia Roldán* \\ Public-Private Sector Research Center, IESE Business School ${ }^{\dagger}$
}

November, 2009

\begin{abstract}
A decision-maker has to elicit information from multiple informed experts about a policy's value. The principal may prevent communication among agents. However, it may be in the principal's interest to allow communication among them. I assume that communication allows some synergies among experts to emerge but also opens the possibility of collusion among them. I study the optimal design of contracts focusing on the organization of expertise in the communication phase. I show that, from principal's point of view, when the advantages of the synergy's effects prevail over the collusion problem, communication dominates no communication. However, this will be always true, when the principal allows agents to communicate with each other before they make their effort choices instead of after.
\end{abstract}

JEL Classification Numbers: D81; D82: L23

Keywords: information acquisition;communication; coordination; collusion; expertise; organization

${ }^{*}$ I am grateful to Antonio Cabrales for his advice, guidance and support. I have greatly benefited from the comments and useful suggestions of Carlos Ponce, Antonio Romero, Guillermo Caruana, José Penalva and Juan José Ganuza. I am also grateful to the participants of the EEA 2009 Annual Meeting in Barcelona, Spain; the EARIE 2009 Annual Meeting in Ljubljana, Slovenia, and the audience of the SP-SP Research Center's Lunch Seminars Series at the IESE Business School. The usual disclaimers apply.

${ }^{\dagger}$ Address for Correspondence: Flavia Roldán: Public-Private Sector Research Center, IESE Business School, Av. Pearson 21, (08034), Barcelona, Spain, E-mail: froldan@iese.edu 


\section{Introduction}

Many decisions in real life are complex and involve a multiplicity of aspects. In these cases decision-makers rarely have the time or the skills to gather, process, and summarize all the relevant information to make better decisions. Therefore, it is usually observed that they base their decisions on the information provided by specialized experts who are hired for that specific goal. In this research, the particular way in which information is produced plays an important role. In particular, as Arrow pointed out (1969, p.30), "Knowledge arises from deliberate seeking, but it also arises from observations incidental on other activities".

More precisely, the goal of this article is to examine the following economic framework. An uninformed principal ("she"), must elicit information from unbiased agents (experts, "he") who must, in turn, decide whether or not to collect costly information (this is for us what Arrows calls "deliberate seeking"). After that, if agents communicate among each other, each one will obtain a more precise signal about the information initially acquired (this is for us Arrows' remark about "Knowledge...also arises from observations incidental on other activities"). Finally, this simple framework also incorporates an opportunistic consideration of communication. I assume that communication opens the possibility of collusion among the agents against the main interest of the principal.

Since communication has conflicting consequences, one question addressed in this article is: Should principals promote or impede communication among experts? Then, if the principal allows communication among experts, what is the best way to organize agents who are in communication? These issues are studied in a multiagent-principal framework when communication among agents allows not only cooperation in favor of the principal but also collusion against her.

In our setting, the principal has to decide whether to implement a policy which has two possible observable outcomes: success or failure. She gains access, through agents, to noisy signals. The signals are independent conditional on the policy's outcome. Each signal has two possible values: "good news" or "bad news" about the policy's success.

Each expert must gather information and send a report to the principal about the true state of the policy. These tasks have special features: gathering information is costly, and since reports are soft information, they are fully manipulable. ${ }^{1}$ Therefore, experts must be given incentives to both gather and truthfully transmit precise information.

We study and compare the principal's net surplus under different organizational forms.

First, I compare the principal's net surplus under the no communication and communication case, but when it only involves its detrimental effect, i.e., potential collusion. In such a case, the principal is better off avoiding communication among agents since communication only imposes additional constraints on her problem.

\footnotetext{
${ }^{1}$ That is, the signal observed by agents and their reports need not coincide. Additionally, experts are protected by limited liability.
} 
Then, I consider the situation in which the principal organize experts in a common workplace. i.e., a team of experts, and thus allows communication among them. In this case, communication has conflicting consequences.

On the one hand, when agents communicate with each other, signals are more precise than in the absence of communication. This fact has not only a positive direct impact on the principal's surplus but it also makes the informational problem less severe. On the other hand, when agents are in communication, they are able to sign contracts contingent on information that is verifiable inside the team, make reports that are jointly optimal for them, and exchange side-transfers.

In contrast, when the principal prevents communication among agents, she sacrifices the signals' precision. In this circumstance, however, the principal takes advantage of multiple reports by cross-checking them.

I find that when the signal's precision is sufficiently increased with communication, the principal will be better off organizing experts in a common workplace, where they are able to exchange their knowledge, rather than avoid communication among them.

Nevertheless, this intuitive result may change as the organization of expertise changes in the communication phase. More precisely, when should the principal allow agents to communicate with each other? Before or after the agents gather information?

When agents are able to communicate with each other before they collect any signal, they are not only able to manipulate the information that they reveal to the principal, but they can also sign side contracts contingent on their decision whether or not each one collects information.

In the case where agents can only communicate with each other after exerting effort, they cannot coordinate their effort choices in collecting information. In such a case, however, each agent makes that decision without information about the other agents' decision. Although the principal can also prevent collusion on the choice of effort in such situations, she imposes more risk on the agents than would be imposed if each expert had knowledge about the effort exerted by the other experts.

Therefore, when communication is permitted, I show that the best way of exploiting the positive effect of communication is to let agents communicate with each other from the outset. In other words, the principal's welfare increases with this coalition among experts. Consequently, when the advantages of the communication (i.e., the increase in the signals' precision) sufficiently outweigh the disadvantages of collusion, the principal is better off allowing the agents to communication with each other from the outset, rather than avoid communication.

The current article is linked to three lines of research: endogenous acquisition of information, transmission of information and organization of expertise.

The literature focusing on information revelation obtains as its main result that if there are no costs of supplying information, perfect information transmission requires that the decision-maker and the expert have identical preferences. In this line of research, Wolinsky (2002) is close to this article. The focus is on how the decision-maker can take advantage of multiple experts. He shows that in some circumstances, allowing partial communication among experts may result in the revelation of more information 
than either full communication or no communication.

However, in all these papers, the focus is on strategic information revelation rather than on information acquisition. In contrast, in the current article, the decision-maker elicits information from multiple unbiased experts, and agents must decide whether or not to acquire information. Several authors analyze this issue in the literature. For example, Li (2001) and Szalay (2005) examine information acquisition when players have the same preferences but it is not possible to implement monetary incentives. Li (2001) assumes that the precision of the signal provided by the agents increases with effort, and he shows that the commitment to excessive conservatism in committee decision-making can be used to increase the incentives to acquire information. Szalay (2005) also considers that the probability to learn the state increases with effort, and he shows that the principal finds it optimal to let the agent choose the action but excludes from the choice those actions that are optimal when no information is acquired. However, as in the current article, Gromb and Martimort (2007), consider the design of monetary incentives, and they study the implications of optimal incentives contracts for the organizational design of expertise. They assume a principal, who bases her decision on two signals about a project's value, and agents, who can draw independent signals at a fixed cost per signal. After receiving the signals, the agents recommend to either undertake the project or not undertake it. They show that it is optimal to reward the agent if his recommendation is confirmed by the state or by another recommendation (conflicting reports are penalized). ${ }^{2}$ Subsequently, they analyze when it is optimal, from the principal's point of view, to have a single expert gathering two signals or two experts collecting one signal each.

In the present paper, unlike Gromb and Martimort (2007), I assume that the signals' precision not only increases with effort (a fixed cost per signal) but also increases with (horizontal) communication among agents. And, at this point, the organization of expertise becomes crucial. With multiple agents, how should experts be organized to ensure that they refine their knowledge about the true state and fully disclose their signals? In the current article, unlike Gromb and Martimort (2007), I analyze the optimal organization of communication among experts. This particular feature is close to Itoh (1993) in that the principal benefits from contracting a consolidated unit whose utility is the sum of its members' utilities and in which employees can monitor each other's effort and coordinate their actions. Nikolova (2005) analyzes the optimal mutual monitoring-incentive pay mix and she finds that the optimal mix depends on the agents' liability limit.

The paper proceeds as follows. Before introducing the model, I highlight the paper's main issues by providing an example. Then, Section 2 presents the general setting, and Section 3 contains an analysis of the optimal organization when communication does not improve the accuracy of the signals obtained by the agents. Section 4 compares the principal's surplus when communication implies not only collusion among experts but

\footnotetext{
${ }^{2}$ Köhler (2004) shows that this does not necessarily hold when the state and signal space are continuous.
} 
also synergistic effects among them. In such a case, I study the optimality, from the principal's point of view, of two alternative organizations of communication. Namely, communication among agents before they decide to collect information, and after they choose whether or not to gather information. Finally, Section 5 gives the conclusions. All proofs and details of the calculations are in the Appendix.

\section{An example: an intelligence problem}

Consider the problem faced by the Director of Intelligence of country A. The Director has received an alert of sabotage against the tabloid press and must decide whether or not to impose a red alert. She hires two spies who must provide information about the likelihood of that sabotage. Let us assume that the spies work in isolation and the agents' identity remains unknown to each other. Each spy must decide whether or not to collect intelligence data. After collecting data, he processes all this information and obtains a noisy signal about the probability of an attack occurring.

For example, one of the spies might obtain information from the interception of communications (telephone calls, e-mails, letters and so on). He processes all this data and he obtains a signal, although some of the individuals mentioned in the telephone calls or in the letters cannot be declared "dangerous" because he has no proofs for that. The other spy concentrates his investigations on studying information about people who have entered and left the country in the past year. Similarly, he processes all this data, gets a signal, but he does not find any conclusive evidence that some particular individuals being investigated are involved in the attack on the yellow press.

The Director of Intelligence receives one signal from each agent, after which he will make a decision. If the spies supply conflicting signals, the Director penalizes them, since the state of the world is only one (i.e., sabotage or not).

However, let us assume, that the Director allows the agents to communicate with each other. In such a case, they would mutually exchange their initial knowledge and certain items of information that before were irrelevant would now become important for the investigation. For example, they would realize that the names of some of the individuals mentioned in the letters or in the telephone calls, (and that, before communication, they were irrelevant for the inquiry) match the people who have entered and left the country in the last few months (and again, before communication, they were impertinent for the investigation), and this coincidence could provide sufficient evidence that these people are involved in the attack. Therefore, communication improves the signals' precision.

Nevertheless, since the spies are in communication, they may coordinate their reports to show that, for example, the sabotage is only a rumor spread by the yellow press itself but without observing it. Then, the Director's problem now is whether or not to allow communication between the spies. If the benefit from sharing information outweighs the potential collusion cost, it seems reasonable that both spies work together as a single intelligence team.

Organization of Communication. How should the spies be organized in the 
communication phase? At what point should the principal allow the agents to communicate with each other?

Before. If the spies' identity is revealed from the outset, they may collude not only on their reports but also in their decision about whether or not to collect information.

After. If each agent knows his partner's identity only after he decides whether or not to gather information, the Director avoids the possibility of collusion on the effort choices, and she still takes advantage of exchange of information between the spies. In such a case, however, each spy makes his effort decision without knowing the other spy's decision.

Consequently, the Director should decide not only whether or not she allows communication but she must also decide the optimal time for allowing the spies to communicate with each other. I find that it is optimal, from the Director's perspective, to reveal the spies' identity from the outset, i.e. before they make their effort choices.

\section{The General Setting}

I consider the relationship between one risk-neutral principal (decision-maker) and $n=$ 2 risk neutral-agents (experts).

The decision-maker has to choose an action $a \in\{0,1\}$, where 1 stands for implementing a policy $y$, and 0 otherwise. The outcome of that policy $y$ is either success or failure, that is $y \in\{0,1\}$. The common prior for success is $\operatorname{Pr}(y=1)=v=1 / 2$.

When the policy is undertaken, it will have two possible observable monetary outcomes; i.e., $S>0$ when $y=1$, or $F<0$ otherwise. If the policy is not undertaken, its outcome will not be observed.

To sum up, the principal's gross payoff depends on $a$ and on an unknown state of the world $y$, according to the following:

$$
V(a, y) \begin{cases}S & \text { if } a=1 \text { and } y=1 \\ F & \text { if } a=1 \text { and } y=0 \\ 0 & \text { otherwise }\end{cases}
$$

We assume that, without additional information, it is not efficient to implement the policy; i.e., $E[V(a, y)]=v S+(1-v) F<0$. Therefore, in such a case, the principal's optimal decision is $a=0$; i.e., the status quo.

The decision-maker, however, has neither the time nor the skills to gather and process all information related to the policy's success. Therefore, she consults unbiased agents or experts, i.e., they respond only to monetary incentives.

Each expert must simultaneously decide whether or not to exert effort; i.e., $e_{i} \in$ $\{0,1\}$. In other words, each one must decide whether to gather and study information about the policy's success, i.e. $e_{i}=1$, or not, i.e., $e_{i}=0$. I assume that exerting effort is costly, therefore $c\left(e_{i}=0\right)=0$ and $c\left(e_{i}=1\right)=c>0$. 
When $e_{i}=1$, expert $i$ gets a noisy signal $\sigma_{i} \in\left\{\underline{\sigma}_{i}, \bar{\sigma}_{i}\right\}$, where $\bar{\sigma}_{i}$ means that the policy's outcome $y$ is more likely to be a success ("good" news), and $\underline{\sigma}_{i}$ increases the likelihood that $y$ may be failure ("bad" news). I assume that noisy signals are independent conditional on the policy's outcome.

The agent's output is a precise report about the true state of the world. Let define the report's precision as $p^{i}(\cdot) \equiv p\left(\bar{\sigma}_{i} \mid y=1\right)=p\left(\underline{\sigma}_{i} \mid y=0\right), \forall i$; and let assume the following:

Assumption $1 p^{i}(\cdot)=p^{i}\left(e_{i}\right) \in(0,1)$, and $p^{i}\left(e_{i}\right)=p^{j}\left(e_{j}\right), \forall i, j$ such that $e_{i}=e_{j}$ with $p^{i}\left(e_{i}=0\right)=v$ and $p^{i}\left(e_{i}=1\right)=a>1 / 2, \forall i$.

The Assumption says that the signal precision of agent $i$ depends on the level of effort supplied by agent $i$. Additionally, I assume that all experts are equally precise, and if an agent chooses "shirk", i.e., $e_{i}=0$, he produces a signal which has a precision equal to the common prior. However, if the expert chooses "work", i.e., $e_{i}=1$, he will get a more precise signal.

After that, and before the agents send the report to the principal, they may communicate with each other. In such a case, there are two forces at play in the communication phase. On the one hand, I assume that communication introduces the possibility of collusion among experts. That is, after the agents accept the contract offered by the principal, they can sign a contract contingent on verifiable information and jointly manipulate the information they obtain in their own interest.

On the other hand, I assume that communication increases the precision of the signal that each agent receives. We can imagine that after agents exert some effort, they have a "rough" idea about the true state of the world. If they were able to communicate this preliminary knowledge with each other, they would obtain a more "refined" idea about the desirability of the principal's actions. We can interpret this as the communication among experts allows that some synergies among them to emerge.

Hence, the communication process produces a signal which has, at least, an equal precision to no communication.

Let us define $E_{-i}=\sum_{-i} e_{-i} \in[0, n-1]$ as the level of effort supplied by the experts who are in communication with expert $i$. In other words, $E_{-i}$ is the number of information sources of agent $i$.

Thus, when communication takes place, the final signal's precision depends not only on $e_{i}$ but also on $E_{-i}$. That is, $p^{i}(\cdot)=p^{i}\left(e_{i}, E_{-i}\right)$. The next assumption describes the relationship between all these variables.

Assumption 2

i) $p^{i}\left(1, E_{-i}\right)>p^{i}\left(0, E_{-i}\right)=v$ for all $E_{-i}$.

ii) $p^{i}\left(e_{i}, E_{-i}^{\prime \prime}\right) \geq p^{i}\left(e_{i}, E_{-i}^{\prime}\right)$ for all $E_{-i}^{\prime \prime}>E_{-i}^{\prime}$ and with strict inequality for $e_{i}=1$. 
iii) $p^{i}\left(e_{i}, E_{-i}^{\prime \prime \prime}\right)-p^{i}\left(e_{i}, E_{-i}^{\prime \prime}\right)<p^{i}\left(e_{i}, E_{-i}^{\prime \prime}\right)-p^{i}\left(e_{i}, E_{-i}^{\prime}\right)$ for all $E_{-i}^{\prime \prime \prime}>E_{-i}^{\prime \prime}>E_{-i}^{\prime}$ and for $e_{i}=1$.

The first part of the Assumption says that the communication is only useful if the experts have an initial idea $\left(e_{i}=1\right)$ of what "they are talking about". When two experts communicate with each other, but at least one of them has no idea about the "subject" of the talk, the conversation is useless and the communication does not improve the signal's precision. That is, $p^{i}\left(0, E_{-i}\right)$ is equal to the common prior.

The second part of the assumption says that the signal's precision is an increasing function in the number of information sources that each agent has. In addition, it is strictly increasing in $E_{-i}$ when the agent puts some effort into collecting information, i.e., $e_{i}=1$. In other words, the marginal productivity of his own effort increases as $E_{-i}$ increases.

And finally, Part iii) states that the signal's precision is a concave function in $E_{-i}$. That is, the marginal productivity of communication $\left(p^{i}\left(e_{i}, E_{-i}^{\prime \prime}\right)-p^{i}\left(e_{i}, E_{-i}^{\prime}\right)\right)$ decreases with the number of sources of information.

On the contrary, if agents do not communicate with each other, the principal avoids the collusion problem but she cannot take advantage of the synergy effects among agents that only emerge when they communicate with each other.

After that, the principal asks the experts to send reports. Given these messages, the principal updates her belief about the future state of $y$ and chooses an action. At the end, the state of the world is realized, the transfers are paid, and payoffs are realized.

Finally, I assume that experts are protected by limited liability and they have the same preferences. Thus, the expert's payoff function is

$$
U(t, e)=t(\cdot)-c\left(e_{i}\right)
$$

where $t(\cdot)$ is the transfer that the agent receives from the principal, which we will discuss further.

The principal's net payoff will be

$$
V(a, y)-\sum_{i} t(\cdot)
$$

It is worth remarking that experts produce soft information which is non-verifiable and fully manipulable. Therefore, the principal must solve two kinds of problem: design a contract such that agents exert effort and also truthfully reveal their private information.

In the following, I assume that two alternative organizational structures can take place. In one of them, agents remain isolated and therefore, no exchange of information can occur. I call this kind of organization an isolated work structure, IWS henceforth. In the second alternative, the experts are able to communicate with each other, and I call that a communication work structure, CWS henceforth. 


\section{The benchmark case: IWS versus CWS without synergy effects}

I will show that if there are no synergy effects between experts when they communicate with each other, then the principal is better off when she avoids communication between them. In other words, when communication does not improve the signals' quality, it only imposes additional constraints on the principal's problem.

We first characterize the optimal contract in each situation. Before introducing the benchmark case, it is useful to present some notation and definitions.

Definition 1 Denote by $p(\sigma)$ the likelihood of $\sigma \in\{\underline{\sigma}, \bar{\sigma}\}$.

For example, when an agent exerts effort, $p(\bar{\sigma})=a v+(1-a)(1-v)$. Likewise, since signals are independent conditional on the policy's outcome, $p(\bar{\sigma} \bar{\sigma})=a^{2} v+$ $(1-a)^{2}(1-v)$.

Definition 2 Let $v(\sigma)$ be the probability of success conditional on $\sigma$.

Therefore, $v(\sigma)=p(y=1 \mid \sigma)$. That is, $v(\sigma)=\frac{p(\sigma \mid y=1) p(y=1)}{p(\sigma)}$.

\subsection{The Isolated Work Structure (IWS)}

We consider now an isolated work structure of experts. That is, we are assuming that agents do not communicate with each other. In turn, this implies that the signal each agent obtains, $\sigma_{i}$, as well as whether they make effort, are not observable by other parties, i.e. neither by the principal nor by the other experts.

The timing is as follows. The principal offers each expert a contract. Each one accepts or rejects it. If he accepts, he decides whether or not to gather information, and then sends a message to the principal. Given these messages, she updates her belief about the future state of $y$ and chooses an action. Finally, the state is realized, the transfers are paid, and payoffs are realized.

\section{Optimal Contracts}

Since signals and efforts are not observable by the principal, the transfers can only be based on reports and on the policy's outcome.

Let us note that the policy is only undertaken by the principal when both reports are positive. This is because after the principal receives either two conflicting or two negative signals and updates her beliefs, the optimal action will be to not undertake the policy, i.e. $v(\underline{\sigma \sigma})<v(\underline{\sigma} \bar{\sigma})=v^{3}$

\footnotetext{
${ }^{3}$ That is, $v(\underline{\sigma \sigma})=p(S \mid \underline{\sigma \sigma})$. By solving that, we get $v(\underline{\sigma \sigma})=\frac{(1-a)^{2} v}{(1-a)^{2} v+a^{2}(1-v)}$, which is less than $v$. Additionally, let us recall that, by assumption, $v S+(1-v) F<0$.
} 
In the case in which the principal chooses the status quo, i.e. $a=0$, the reports are not verifiable. However, we will see that the principal can use the correlation between messages to extract informational rents from experts.

Thus, $\bar{t}$ is the transfer received by the expert if the policy is undertaken and it is a success, and $\underline{t}$ is the transfer when the policy is undertaken but it fails. However, if the policy is implemented, $t_{0}$ is the transfer that each agent receives when both signals are negative. In the event of conflicting reports, the expert reporting $\bar{\sigma}$ receives $t_{g}$, and the other expert, whose report is $\underline{\sigma}$, receives $t_{b}$.

The contract that the principal offers must provide agents with incentives to gather information and report it accurately.

A similar problem is solved by Gromb and Martimort (2007), henceforth GM, and for brevity I only report optimal transfers and compute the agency cost for this organizational structure.

The optimal transfers are

$$
\begin{gathered}
\underline{t}=t_{b}=t_{g}=0 \\
\bar{t}=\frac{p(\underline{\sigma}) c^{v}}{v(1-v)(2 a-1) a^{2}} \text { and } t_{0}=\frac{c}{(1-v)(2 a-1) a}
\end{gathered}
$$

while the agency cost for the isolated work structure is $\left(T_{I W S}\right)$

$$
T_{I W S}=2 \frac{c[p(\underline{\sigma})+(1-v)(2 a-1) a]}{(1-v)(2 a-1) a}
$$

In this circumstance, as GM point out, a positive report is only rewarded when the other report is also positive and the policy's outcome is success $\left(\underline{t}=t_{g}=0\right)$. Additionally, conflicting reports are penalized. Since signals are correlated, a negative report is only rewarded when the other report is also negative $\left(t_{b}=0\right)$.

Because of the current principal's ability to cross-check reports, the expert's informational rent is reduced and the agency cost is less than if there were a single agent gathering two signals. ${ }^{4}$

\subsection{The Communication Work Structure (CWS) without syn- ergy effects}

I now assume that after agents exert some level of effort to gather information, they can communicate with each other. By now, I assume that when communication takes place, agents can jointly manipulate their reports in their own interest. The key point here is that communication allows agents to sign self-enforcement contracts contingent on observable variables. ${ }^{5}$

\footnotetext{
${ }^{4}$ Gromb and Martimort (2007) say that in this case, there are "economies of scale due to the agency costs".

${ }^{5}$ Our context assumes that there are no frictions among agents. This differs from Jeon, D.-S and Menicucci, D. (2005). They study the optimal sale mechanism which takes into account not only
} 
In this case, the optimal contract is also based on reports and on the policy's outcome. Although moral hazard constraints are the same as when no communication takes place, adverse selection constraints change and they reflect the principal's inability to distinguish all pairs of reports leading to the status quo. That is, communication, in this circumstance, only introduces additional constraints on the principal's problem.

In this case, the agency cost $\left(T_{C W S}\right)$ is

$$
T_{C W S}=2 c\left[\frac{[1+(1-v)(2 a-1)]}{(1-v)(2 a-1) p(\underline{\sigma})}\right]
$$

and $\bar{t}, t_{0}, t_{b}, t_{g}$ are strictly greater than zero. ${ }^{6}$

\subsection{IWS versus CWS without synergy effects: The cost of collusion}

As we see from the above discussion, the principal can provide a contract which is robust to collusion between experts. However, doing that is costly.

That is, when the communication between experts is not possible, i.e., IWS, the principal avoids collusion problems, and she has the ability to cross-check reports and penalize conflicting reports.

On the contrary, when agents are able to communicate with each other, a coalition or a group of experts must be given incentives to truthfully reveal the information they obtain.

Thus, whether the principal must allow or avoid communication between agents depends on whether the agency's cost of IWS is greater or lesser than the agency's cost of CWS.

By simple manipulation, it is possible to see that

$$
T_{C W S}-T_{I W S}=\frac{(2 a-1) v+p(\bar{\sigma})[p(\underline{\sigma})+a(2 a-1)(1-v)]}{a(2 a-1)(1-v) p(\underline{\sigma})}>0
$$

We can define this difference as the cost of collusion and characterize that by providing some intuition of it.

First of all, let us note that as $v$ increases, the collusion's cost also increases. That is, as the prior of success increases, agents are more tempted to report good news $(\bar{\sigma} \bar{\sigma})$. Consequently, they must be given more incentives to report bad news when they really observe it, i.e., $t_{0} \geq v \bar{t}$.

individual incentive compatibility but also coalition incentive compatibility. They show that, although marginal rates of substitution are not equalized across different types of buyers in the optimal sale mechanism, they fail to realize the gains from arbitration because of the transaction costs in coalition formation generated by asymmetric information.

${ }^{6}$ See Gromb and Martimort (2007). 
Second, let us observe that the cost of collusion is decreasing in $a \in(1 / 2,1] .^{7}$ This is because, when agents are more precise, it is easier for the principal to give experts incentives to report good news when they observe it, i.e., $v(\bar{\sigma} \bar{\sigma}) \bar{t}=\frac{a^{2} v}{p(\bar{\sigma} \bar{\sigma})} \bar{t} \geq t_{0}$.

The following Proposition arises immediately from the above discussion.

Proposition 1 Assumes communication only involves the possibility of collusion among agents and there are no synergy effects. The principal is better off avoiding communication among agents, since the agency cost of IWS is less than the agency cost of CWS.

\section{The organization of communication work struc- ture with synergy effects}

I now analyze the principal's problem when the principal has to elicit information from unbiased experts and, at the same time, she wants to exploit the synergy effects between them that emerge when they are in communication.

I assume that when agents communicate with each other, there are two opposing forces at work. On the one hand, communication allows some synergies between experts to emerge. I assume that experts are working together and this common 'workplace' allows them to exploit some complementarities.

On the other hand, communication between experts has another effect. Experts can jointly manipulate their reports in their own interest. Therefore, communication allows agents to collude against the principal, and hence they may make decisions that improve their welfare but not necessarily the well-being of the principal.

In this environment, I will study, from principal's point of view, which is the best way to exploit the complementarities among experts. To be more precise, I will answer when it is optimal, from principal's point of view, to allow agents to communicate with each other.

\subsection{Communication between experts after exerting effort}

Let us assume that the principal organizes the experts in a common workplace. Thus, they are able to communicate with each other.

Each agent simultaneously decides whether to work or shirk, i.e., gather information or not. If he decides to exert effort, he obtains a signal $\sigma_{i} \in\left\{\underline{\sigma}_{i}, \bar{\sigma}_{i}\right\}, \forall i=1,2$, satisfying $p\left(\sigma_{i}=\bar{\sigma}_{i} \mid y=1\right)=p\left(\sigma_{i}=\underline{\sigma}_{i} \mid y=0\right)=a, \forall i$, where $a \in(1 / 2,1]$. The signals that each expert captures are conditionally independent.

After gathering information, experts can communicate with each other without cost. The communication stage delivers signals, such that $p\left(\sigma_{i}=\bar{\sigma}_{i} \mid y=1\right)=p\left(\sigma_{i}=\underline{\sigma}_{i} \mid y=0\right)=$ $\epsilon, \forall i$, where $\epsilon>a \in(1 / 2,1]$.

\footnotetext{
${ }^{7}$ The collusion cost goes to infinity as $a$ goes to $1 / 2$, and it goes to $\frac{v(1+2(1-v))}{(1-v)^{2}}<\infty$ when $a$ tends to 1 .
} 
After the communication phase, the principal asks agents for a joint report $\sigma_{A} \in$ $\left\{\underline{\sigma}_{A}, \bar{\sigma}_{A}\right\}$, where $A$ refers to an alliance or a group of experts, according to the following: (i) if each expert obtains $\bar{\sigma}$, they must report $\bar{\sigma}_{A}$, or (ii) the group of experts reports $\underline{\sigma}_{A}$ otherwise. This is because $v(\underline{\sigma \sigma})<v(\underline{\sigma} \bar{\sigma})=v$. That is, after the principal receives two negative or conflicting messages, she will always decide the status quo. ${ }^{8}$

After receiving the report, the principal updates her belief about the future state of $y$ and chooses an action. Finally, the state is realized, the transfers are paid, and payoffs are realized.

It is worth emphasizing the information available to each agent in each phase:

i Whether or not the expert gathers information is not observable either by the principal or by the other group's members.

ii The signal $\sigma_{i}$ is not observable by the principal but it is observable by other experts in the communication phase.

Then, as before, transfers are only based on reports and on the policy's outcome. Thus, when experts report $\bar{\sigma}_{A}$, the policy is undertaken and each expert receives $\bar{t}$ if the policy is a success. ${ }^{9}$ When experts report $\underline{\sigma}_{A}$, the policy is not undertaken, and each agent receives $t_{0}$.

\section{Optimal Contracts}

The principal must provide incentives to each expert to acquire information and also reveal it truthfully.

Moral hazard incentive constraints on gathering information are such that each expert will not prefer to remain uninformed and report $\underline{\sigma}$ :

$$
p\left(\bar{\sigma}_{A}\right) v\left(\bar{\sigma}_{A}\right) \bar{t}+\left(1-p\left(\bar{\sigma}_{A}\right)\right) t_{0}-c \geq t_{0}
$$

or $\bar{\sigma}$, that is,

$$
p\left(\bar{\sigma}_{A}\right) v\left(\bar{\sigma}_{A}\right) \bar{t}+\left(1-p\left(\bar{\sigma}_{A}\right)\right) t_{0}-c \geq p(\bar{\sigma}) v(\bar{\sigma}) \bar{t}+p(\underline{\sigma}) t_{0}
$$

Additionally, when experts, who are in communication, observe $\bar{\sigma} \bar{\sigma}$, they should not prefer to report $\underline{\sigma}_{A}$ :

$$
2 v\left(\bar{\sigma}_{A}\right) \bar{t} \geq 2 t_{0}
$$

and, when they observe $\underline{\sigma \sigma}$ or $\underline{\sigma} \bar{\sigma}$, they should prefer to report that rather than $\bar{\sigma}_{A}$

$$
2 t_{0} \geq 2 v \bar{t}^{10}
$$

\footnotetext{
${ }^{8}$ Recall that, by assumption, $v S+(1-v) F<0$.

${ }^{9}$ Gromb and Martimort (2007) show that $\underline{t}=0$ is optimal in our context.

${ }^{10}$ Recall that $v(\underline{\sigma \sigma})<v(\underline{\sigma} \bar{\sigma})=v$.
} 
The other constraints on the principal's problem are: (i) the incentive participation constraint for each group's member,

$$
p\left(\bar{\sigma}_{A}\right) v\left(\bar{\sigma}_{A}\right) \bar{t}+\left(1-p\left(\bar{\sigma}_{A}\right)\right) t_{0}-c \geq 0
$$

and (ii) the limited liability constraints

$$
\bar{t}, t_{0} \geq 0
$$

Then, the principal's program is:

$$
\min p\left(\bar{\sigma}_{A}\right) v\left(\bar{\sigma}_{A}\right) \bar{t}+\left(1-p\left(\bar{\sigma}_{A}\right)\right) t_{0}
$$

subject to (3)-(8).

Lemma 1 The optimal transfers are

$$
\bar{t}=\frac{c}{2 v(1-v)(\epsilon-a)} \quad \text { and } \quad t_{0}=\frac{c}{2(1-v)(\epsilon-a)}
$$

Likewise, the agency cost when communication takes place after agents exert effort $T_{C W S(F)}$ is

$$
T_{C W S(F)}=\frac{c[1+(1-v)(2 \epsilon-1)]}{(1-v)(\epsilon-a)}
$$

The principal's net surplus is

$$
\epsilon^{2} v S+(1-\epsilon)^{2}(1-v) F-T_{C W S(F)}
$$

In this case, agents are always better off by gathering information rather than not exerting effort and reporting bad news. Additionally, as we can see from (9), when the communication increases the signal's precision the agency cost decreases.

\subsection{Communication between experts before exerting effort}

I now introduce some changes to the expert's organization described in the preceding section. First, the principal organizes the agents in a common workplace and in this common workplace, they are able to observe each other from the outset. That is, each expert knows not only the signal received by the other agents but also whether or not the other agent exerts effort.

Second, as before, the principal offers a contract to each agent, and the team of experts must produce a joint report $\sigma_{A} \in\left\{\underline{\sigma}_{A}, \bar{\sigma}_{A}\right\}$, such that the group reports $\bar{\sigma}_{A}$ when each expert obtains $\bar{\sigma}$, and $\underline{\sigma}_{A}$ otherwise. 
Given this message, the principal updates her belief about the future state of $y$ and chooses an action. Finally, the state is realized, the transfers are paid, and payoffs are realized.

Therefore, the information available to each agent in each phase is the following:

i Whether or not the expert gathers information is not observable by the principal but it is observable by the other group's members.

ii The signal $\sigma_{i}$ is not observable by the principal but it is observable by the other experts in the communication phase.

Transfers are based on reports and on the policy's outcome.

\section{Optimal Contracts}

The principal must provide incentives to the alliance or group of agents to acquire information and also reveal it truthfully.

In this case, moral hazard incentive constraints on gathering information are such that the group of agents will not prefer its members to remain uninformed and report either $\underline{\sigma}_{A}$ or $\bar{\sigma}_{A}$. Therefore:

$$
2\left[p\left(\bar{\sigma}_{A}\right) v\left(\bar{\sigma}_{A}\right) \bar{t}+\left(1-p\left(\bar{\sigma}_{A}\right)\right) t_{0}-c\right] \geq \max \left\{2 t_{0}, 2 v \bar{t}\right\}
$$

Moreover, the team should not prefer to base its report on only one signal. Thus,

$$
2\left[p\left(\bar{\sigma}_{A}\right) v\left(\bar{\sigma}_{A}\right) \bar{t}+\left(1-p\left(\bar{\sigma}_{A}\right)\right) t_{0}-c\right] \geq 2\left[p(\bar{\sigma}) v(\bar{\sigma}) \bar{t}+(1-p(\bar{\sigma})) t_{0}\right]-c
$$

Likewise, the adverse selection constraints are the following two. If each team's member observes $\bar{\sigma}$, the team should prefer to report $\bar{\sigma}_{A}$ rather than $\underline{\sigma}_{A}$. That is:

$$
2 v\left(\bar{\sigma}_{A}\right) \bar{t} \geq 2 t_{o}
$$

If alliance members observe either $\underline{\sigma \sigma}$ or $\underline{\sigma} \bar{\sigma}$, the alliance should prefer to report $\underline{\sigma}_{A}$ rather than $\bar{\sigma}_{A}$. That is:

$$
2 t_{o} \geq 2 v \bar{t}^{11}
$$

The incentive participation constraint for each team's members is

$$
p\left(\bar{\sigma}_{A}\right) v\left(\bar{\sigma}_{A}\right) \bar{t}+\left(1-p\left(\bar{\sigma}_{A}\right)\right) t_{0}-c \geq 0 .
$$

and the limited liability constraints are

$$
\bar{t} \geq 0, t_{0} \geq 0
$$

\footnotetext{
${ }^{11}$ See footnote 8.
} 
Hence, the principal's program is:

$$
\min p\left(\bar{\sigma}_{A}\right) v\left(\bar{\sigma}_{A}\right) \bar{t}+\left(1-p\left(\bar{\sigma}_{A}\right)\right) t_{0}
$$

subject to (10)-(15).

Lemma 2 When $\epsilon-a>a-\frac{1}{2}$, the optimal transfers are

$$
\bar{t}=\frac{c}{v(1-v)(2 \epsilon-1)} \quad \text { and } \quad t_{0}=\frac{c}{(1-v)(2 \epsilon-1)}
$$

and, in this case, the agency's cost $\left(T_{C W S(B)}\right)$ is

$$
T_{C W S(B)}=2\left[\frac{c(1+(1-v)(2 \epsilon-1))}{(1-v)(2 \epsilon-1)}\right]
$$

The principal's net surplus is

$$
\epsilon^{2} v S+(1-\epsilon)^{2}(1-v) F-T_{C W S(B)}
$$

When $\epsilon-a>a-\frac{1}{2}$, the team of experts is always better off collecting $n=2$ signals rather than only one. Therefore, if each agent in the group is better off collecting one signal each, then each one will be better off reporting the truth, otherwise it would not pay for these signals. Hence, the adverse selection problem is implied by the moral hazard problem.

The agency's cost expression is intuitive. We can rewrite it as:

$$
T_{C W S(B)}=2\left[\frac{c(1+(1-v)(2 \epsilon-1))}{\left[p\left(\bar{\sigma}_{A} \mid y=1\right)-p\left(\bar{\sigma}_{A}\right)\right]}\right]
$$

First recall that $p\left(\bar{\sigma}_{A} \mid y=1\right)=\epsilon^{2}$. Therefore, as $\epsilon$ increases due to communication, it is less costly to induce agents to reveal good news $(\bar{\sigma} \bar{\sigma})$ when they observe it. That is, constraint (12) is relaxed, and in turn this also relaxes the moral hazard constraints. Thus, the agency's cost reduces as $\epsilon$ increases.

In contrast, as $v$ increases, $p\left(\bar{\sigma}_{A}\right)$ increases, and it is easier to correctly guess "good news" without observing it. Thus, constraint (13) is tightened. In turn, it implies that the moral hazard problem is more severe, hence, the agency cost will be higher.

Lemma 3 When $\epsilon-a<a-\frac{1}{2}$, the optimal transfers are

$$
\bar{t}=\frac{c}{4 v(1-v)(\epsilon-a)} \quad \text { and } \quad t_{0}=\frac{c}{4(1-v)(\epsilon-a)}
$$

and, in this case, the agency's cost $\left(T_{C W S(B)}\right)$ is

$$
T_{C W S(B)}=\frac{c(1+(1-v)(2 \epsilon-1))}{2(1-v)(\epsilon-a)}
$$


The principal's net surplus is

$$
\epsilon^{2} v S+(1-\epsilon)^{2}(1-v) F-T_{C W S(B)}
$$

When $\epsilon-a<a-\frac{1}{2}$, the group of agents is always better off by collecting information, but the principal must give incentives to experts to base the report on two signals rather than only one. As the communication increases signal precision, i.e. as $(\epsilon-a)$ increases, it is less costly for the decision-maker to induce the agents to tell the truth.

\subsubsection{Communication: before or after?}

In the present context, the principal, who exploits the synergy effects between experts, allows communication between them. However, when agents are in communication, they may collude against the principal. That is, experts may share in a credible way their information, make a report that is jointly optimal for them and after that, exchange side-transfers. ${ }^{12}$

We may interpret this as follow. Exploiting the synergy between agents bears some cost in terms of collusion. Consequently, is there any way to reduce that cost?

When the principal allows agents to communicate with each other before exerting effort the principal is also allowing them to write a side-contract contingent not only on the signal and the policy's outcome, but also on their effort choices. Consequently, the principal offers experts a contract such that the team (the coalition) of agents chooses an effort pair which is optimal from principal's point of view.

On the other hand, when communication is only possible after agents have exerted effort each agent selects his own effort without knowing the other expert's choice. In other words, in this case, agents are not able to coordinate their selection of effort.

Proposition 2 In the presence of communication and synergy effects between experts, the principal is better off by allowing the experts to communicate before they collect information rather than after they exert effort.

Proof. By simple manipulation, it is easy to check that $T_{C W S(F)}-T_{C W S(B)}>0$, regardless whether $\epsilon-a \lesseqgtr a-1 / 2$.

In other words, the principal is better off allowing agents to coordinate with each other on their effort choices rather than no coordination at all. Let us observe that, in both cases, i.e., before and after exerting effort, the coalition of agents can manipulate the report sent to the principal. Consequently, in both cases, the team of experts must be given incentives to reveal the truth. However, when the agents are able to communicate from the outset, they are able to select an effort pair which they optimally agree on.

\footnotetext{
${ }^{12}$ I assume enforcable side-contract between experts.
} 
The principal imposes more risk on the agents when she allows them to communicate only after collecting information rather than before gathering information. We can follow that by comparing inequalities (3) and (4) with (10).

Therefore, it is this coordination among experts in selecting effort that reduces the agency cost of communication.

\subsection{IWS versus CWS with synergy effects}

At this point, the question is: Is the principal better off allowing agents to communicate with each other or not allowing them?

When agents communicate with each other, signals are more precise than when no communication exists. This fact has not only a positive direct impact on the principal's surplus but it also makes the informational problem less severe. Additionally, when communication between agents exists and experts are organized into a team of experts from the outset, they are able to coordinate their efforts. That is, the principal imposes less risk on the experts because in this case, the group's members can observe each other and therefore they are able to coordinate their effort choices in collecting information. Hence, the coordination between agents reduces the agency cost.

On the other hand, when no communication exists, the principal sacrifices precision but she can take advantage of multiple reports and penalize conflicting messages.

Proposition 3 When $\epsilon-a>a-1 / 2$, the principal is better off allowing communication between agents before they decide whether or not collect information rather than not allowing any communication at all. When $\epsilon-a<a-1 / 2$, the principal may be better off by allowing communication from the outset.

Proof. In the Appendix.

When $\epsilon-a>a-1 / 2$, we can easily show that $T_{I W S}>T_{C W S(B)}$. When $\epsilon-a<a-1 / 2$, the relationship between agency costs is reversed and $T_{I W S}<T_{C W S(B)}$. Therefore, the principal will be better off with communication if it sufficiently increase the principal's gross payoff. ${ }^{13}$

Therefore, if the signal's precision increases sufficiently with communication, the principal always prefers to allow communication between experts and to organize them as a team from the outset rather than not allow communication at all.

\section{Conclusion}

The aim of this article is to understand how the organization of expertise affects the production and transmission of accurate information by taking into account the incentive problems that may arise. This issue is studied in a multiagent-principal framework

\footnotetext{
${ }^{13}$ That is, if $\left[\epsilon^{2} v S+\left(1-\epsilon^{2}\right)(1-v) F\right]-\left[a^{2} v S+\left(1-a^{2}\right)(1-v) F\right]$ sufficiently compensates the difference $T_{I W S}-T_{C W S(B)}$.
} 
when communication among agents has conflicting consequences. On the one hand, I assume that communication allows some synergies between agents to emerge. On the other hand, communication also allows agents to collude among each other. We concentrate on an uninformed principal who has to elicit information from unbiased experts. I study the optimal design of contracts in different communication settings and I focus on the organization of the expertise, specially in the communication phase. Experts must decide whether to acquire costly information, and after that, if the communication stage takes place, they send an aggregate report to the principal. In contrast, if there is no communication among experts, each one sends the principal one individual report.

Accordingly, if the principal organizes experts such that communication is not possible, she avoids the collusion problem but she cannot take advantage of the cooperation between agents. If communication takes place, it is better for the principal to form a team of experts from the outset. This kind of organization enables the principal not only to exploit the synergy effects among experts but also takes advantage of the coordination of their effort choices. Thus, when the advantages of the synergy effects outweigh the disadvantages of collusion, only horizontal communication from the outset improves the principal's welfare.

This article suggests some interesting avenues for research. One of them arises when we ask the following question: what happens if synergies vary between agents? When synergies between agent $\mathrm{A}$ and agent $\mathrm{B}$ differ from synergies between agent $\mathrm{A}$ and agent $\mathrm{C}$, what is the optimal organization of expertise from the principal's point of view?

Another interesting, and more realistic, avenue to study emerges when we relax the assumption about the cost of communication. Up to now, we assume horizontal communication is costless. When we assume that communication among agents is costly (for example, communication is time-consuming; sometimes it is not easy to "translate" certain specific knowledge for an expert who has different skills, and so on), experts must be given incentives not only to gather costly initial information but also

to communicate among themselves. Consequently, what is the optimal organization of expertise from the principal's point of view?

\section{Appendix}

\section{Proof Proposition 1}

By simple manipulation, we obtain

$$
T_{C W S}-T_{I W S}=2 c\left[\frac{(2 a-1) v+p(\bar{\sigma})[a(2 a-1)(1-v)+p(\underline{\sigma})]}{a(2 a-1)(1-v) p(\underline{\sigma})}\right]>0
$$

\section{Proof Lemma 1}

Assume that $t_{0}=v \bar{t}$, then (5) is slack.

Since $t_{0}=v \bar{t}$, then RHS of (4) is greater than RHS of $(3)$.

Assume that $p\left(\bar{\sigma}_{A}\right) v\left(\bar{\sigma}_{A}\right) \bar{t}+\left(1-p\left(\bar{\sigma}_{A}\right)\right) t_{0}-c=p(\bar{\sigma}) v(\bar{\sigma}) \bar{t}+p(\underline{\sigma}) t_{0}$

After simple manipulation, we get the transfers and the agency cost that are in the 
text.

At this level of transfers, we can check that the other inequalities hold.

Proof Lemma 2

Assume that $t_{0}=v \bar{t}$, then (12) is slack. Likewise, assume that (10) holds with equality and (11) is slack.

By simple manipulation, when $\epsilon-a>a-1 / 2$, we find the transfers and agency costs that are in the Lemma.

\section{Proof Lemma 3}

Assume that $t_{0}=v \bar{t}$, then (12) is slack. Likewise, assume that (11) holds with equality and (10) is slack.

By simple manipulation, when $\epsilon-a<a-1 / 2$, we find the transfers and agency costs that are in the Lemma.

\section{Proof Proposition 2}

Assume $\epsilon-a<a-1 / 2$. In such a case, it is easy to show that $T_{C W S(A)}>T_{C W S(B)}$. That is,

$$
\frac{c(1+(1-v)(2 \epsilon-1))}{(1-v)(\epsilon-a)}>\frac{c(1+(1-v)(2 \epsilon-1))}{2(1-v)(\epsilon-a)}
$$

When $\epsilon-a>a-1 / 2$, we can also show that $T_{C W S(A)}>T_{C W S(B)}$. That is,

$$
\frac{c(1+(1-v)(2 \epsilon-1))}{(1-v)(\epsilon-a)}>2\left[\frac{c(1+(1-v)(2 \epsilon-1))}{(1-v)(2 \epsilon-1)}\right]
$$

Since in these cases the gross payoff for the principal is the same $\left(\epsilon^{2} v S+(1-\epsilon)^{2}(1-v) F\right)$, the principal will be always better off allowing agents to communicate with each other before they exert effort.

\section{Proof Proposition 3}

When $\epsilon-a>a-1 / 2$, we find that $T_{I W S}>T_{C W S(B)}$. That is,

$$
2\left[\frac{c[p(\underline{\sigma})+(1-v)(2 a-1) a]}{(1-v)(2 a-1) a}\right]>2\left[\frac{c(1+(1-v)(2 \epsilon-1))}{(1-v)(2 \epsilon-1)}\right]
$$

Since the gross payoff for the principal when experts work in communication is greater than the gross payoff for the principal when they work in isolation i.e. $\epsilon^{2} v S+$ $(1-\epsilon)^{2}(1-v) F>a^{2} v S+(1-a)^{2}(1-v) F$, the principal always prefers to allow agents to communicate with each other and from the outset.

However, when $\epsilon-a<a-1 / 2$, we can show that $T_{I W S}<T_{C W S(B)}$. That is,

$$
2\left[\frac{c[p(\underline{\sigma})+(1-v)(2 a-1) a]}{(1-v)(2 a-1) a}\right]>\frac{c(1+(1-v)(2 \epsilon-1))}{2(1-v)(\epsilon-a)}
$$

Therefore, if $\left[\epsilon^{2} v S+(1-\epsilon)^{2}(1-v) F\right]-\left[a^{2} v S+(1-a)^{2}(1-v) F\right]$ sufficiently compensates the difference in the agency cost, the principal will be better off by allowing agents to communicate with each other from the outset. 


\section{References}

[1] Arrow, K.,1969. Classificatory Notes on the Production and Transmission of Technological Knowledge. The American Economic Review 59, 29-35.

[2] Bergemann, D. and Välimäki, J., 2007. Information in Mechanism Design. Mimeo, Cowles Foundation Yale University, Discussion Paper $\mathrm{N}^{o} 1208$.

[3] Cai, H., 2003. Costly Participation and Heterogenous Preferences in Information Committees. Mimeo, UCLA

[4] Crawford, V. and Sobel, J., 1982. Strategic Information Transmission. Econometrica $50,1431-51$.

[5] Demski, J. and Sappington, D., 1987. Delegated Expertise. Journal of Accounting Research 25, 68-89.

[6] Dewatripont, M. and Tirole, J., 1999. Advocates. Journal of Political Economy $117,1-41$.

[7] Dur, R. and Swank, O., 2005. Producing and manipulating information. Economic Journal 115, 185- 199.

[8] Gromb, D and Martimort, D., 2007. Collusion and the organization of delegated expertise. Journal of Economic Theory 137, 271-299.

[9] Gerardi, D. and Yariv, L., 2007. Information Acquisition in Committees. Mimeo, Cowles Foundation Discussion Paper 1411R.

[10] Goldfayn, E., 2006. Organization of R\&D with Two Agents and Principal. Mimeo, Bonn ECon Discussion Paper 3/2006.

[11] Holmström, B., 1982. Moral Hazard in Teams. The Bell Journal of Economics 13, 324-340.

[12] and Milgrom, R., 1990. Regulating Trade Among Agents. Journal of Institutional and Theoretical Economics 146, 85-105.

[13] Itoh, H., 1991. Incentive to Help in Multi-Agent Situations. Econometrica 13, 611636.

[14] - 1993. Coalitions, Incentives and Risk Sharing. Journal of Economic Theory $60,410-427$.

[15] Jeon, D.-S and Menicucci,D., 2005. Optimal Second-degree Price Discrimination and Arbitrage: On the role of Asymmetric Information among Buyers. Rand Journal of Economics 36, 337-360. 
[16] Köhler, W., 2004. Optimal Incentive Contracts for Experts. Mimeo, Bonn ECon Discussion Paper 6/2004.

[17] Krishna,V. and Morgan, J., 2001. A Model of Expertise. The Quarterly Journal of Economics 16, 747-775.

[18] Laffont, J.-J and Martimort, D., 1997. Collusion under Asymmetric Information. Econometrica 65, 875-911.

[19] , 2000. Mechanism Design with Collusion and Correlation. Econometrica 68, 309-342.

[20] Li, H., 2001. A Theory of Conservatism. Journal of Political Economy 109, 617-636.

[21] Macho-Stadler, I. and Pérez-Castrillo, J.D., 1993. Moral hazard with several agents. The gains from cooperation. International Journal of Industrial Organization 11, 73-100.

[22] Mukhopadhaya, K., 2003. Jury Size and the Free Rider Problem. Journal of Law, Economics and Organization 19, 24-44.

[23] Nikolova, R., 2005. Mutual Monitoring versus Incentive Pay in Teams. Mimeo, CREST-LEI and LASER-LAEC.

[24] Ottaviani, M. and Sorensen, P.N., 2004. Professional Advice. Mimeo, London Business School and University of Copenhagen.

[25] Osband, K.,1989. Optimal Forecasting Incentives. Journal of Political Economy 97,1091-1112.

[26] Persico, N., 2004. Committee Design with Endogenous Information. Review of Economic Studies 71, 165-191.

[27] Szalay, D., 2005. The Economics of Clear Advice and Extreme Options. Review of Economic Studies 72, 1173-1198

[28] Szalay, D. and Arean, R. ,2005. Communicating with a Team of Experts. Mimeo,.HEC Lausanne and FAME.

[29] Wolinsky, A., 2002. Eliciting Information from Multiple Experts. Games and Economic Behavior 41, 141-160. 